\title{
RELAJACIÓN MUSCULAR CON SUCCINILCOLINA SUBLINGUAL
}

\author{
Erika M. Cuevas, $M D^{*}$, Luis E. Reyes, $M D^{* *}$, José A. Dussich, $M D^{*}$, \\ Sandra H. Martinez, MD*, Alvaro Tito Jiménez, MD*, Carlos DiazGranados, MD***
}

\section{Resumen}

Objetivo: determinar la utilidad de la succinilcolina aplicada por vía sublingual como relajante muscular. Esta información es relevante para extrapolarla a situaciones clínicas en las que por razones técnicas se dificulta la administración intravenosa.

Métodos: se diseñó un ensayo clínico controlado, en el que pacientes adultos de bajo riesgo (ASA I y II) se asignaban para recibir succinilcolina sublingual (SSL) $(1 \mathrm{mg} / \mathrm{k})$, intramuscular (SIM) $(5 \mathrm{mg} / \mathrm{k}) 0$ intravenosa (SIV) $(1 \mathrm{mg} / \mathrm{k}) \mathrm{durante}$ la inducción anestésica. La relajación muscular se midió utilizando el estimulador de contracción muscular TOF watch ${ }^{\circledR}$. Para cada paciente se determinaron mediante cronómetro los tiempos de latencia, acción y recuperación. Se utilizaron la prueba de Mann-Whitney para comparar los tiempos, la prueba exacta de Fisher para las frecuencias y el software SAS versión 8.2 para realizar los análisis estadísticos.

Resultados: se escogieron cinco pacientes en forma aleatoria en cada grupo. A pesar del número reducido se decidió suspender la admisión de nuevos casos, ya que se encontraron diferencias significativas muy temprano. El tiempo de latencia fue significativamente menor en los pacientes en quienes se utilizó SIV en comparación con SIM y SSL. EI tiempo de acción fue más prolongado en quienes se aplicó SIV comparado con aquellos con SIM sin que la diferencia alcanzara significancia estadística. Solo un paciente de los que recibieron SSL alcanzó un tiempo de latencia inferior a diez minutos y un tiempo de acción superior a dos minutos, en comparación con el 100\% de los pacientes que recibieron SIM o SIV $(p=0.02)$. Conclusión: la SSL a una dosis de $1 \mathrm{mg} / \mathrm{k}$ no es útil como relajante muscular. Se requieren más estudios que exploren dosis diferentes para determinar la utilidad clínica de la succinilcolina aplicada por esta vía.

Palabras clave: succinilcolina lingual, laringoespasmo, relajación muscular, adultos.

\section{Introducción}

Una de las complicaciones más temidas de la inducción anestésica es la ocurrencia de laringoespasmo, que consiste en un cierre poderoso y tónico de las cuerdas vocales en respuesta a estímulos variados, que de no tratarse de inmediato puede desencadenar hipoxemia severa, bradiarritmias y paro cardíaco. ${ }^{1}$ Una de las opciones terapéuticas más útiles es el uso

Residente III de Anestesiología, Fundación Universitaria de Ciencias de la Salud, Hospital de San José.

* Autor corresponsal. Coordinador de Investigación Servicio de Anestesiología Hospital de San José. Profesor Asistente Fundación Universitaria de Ciencias de la Salud.

*** Autor corresponsal. Coordinador de Investigaciones Facultad de Medicina, Profesor Asistente Fundación Universitaria de Ciencias de la Salud. de succinilcolina, un relajante muscular de rápido inicio de acción y corta duración. ${ }^{2}$ En el evento de laringoespasmo, la succinilcolina intravenosa (SIV) permite prevenir los desenlaces mencionados, aún cuando se administre a dosis bajas. ${ }^{3}$ Es desafortunado que no siempre el paciente tiene una vía venosa de acceso en el momento del laringoespasmo. Lo anterior sobre todo en niños en quienes la inducción se practica con anestesia inhalada previa a la inserción de un catéter venoso. En estas circunstancias existen reportes anecdóticos en la literatura que mencionan como alternativas el uso de sedantes como el propofol por vía intravascular en los vasos femorales, ${ }^{4}$ el uso de succinilcolina por vía intraósea ${ }^{5,6}$ y el uso de succinilcolina intramuscular (SIM). ${ }^{5,6,7}$ Sin embargo, 
todas estas alternativas presentan dificultades: la vía intravascular femoral es inconveniente para un niño cianótico, la intraósea puede complicarse por extravasación, síndrome compartimental y osteomielitis, y la intramuscular se ha asociado con tiempos de instauración lenta y de acción prolongada. ${ }^{8}$ Hay algunos informes aunque escasos, del uso de succinilcolina sublingual (SSL) o intralingual..$^{9,10}$ Estas opciones son de fácil acceso y presentan estructuras musculares con rica irrigación que permiten suponer en teoría una repuesta más predecible y rápida que cuando se usa como inyección intramuscular convencional. Según la literatura, la dosis utilizada por vía sub o intralingual ha sido de 1 a $3 \mathrm{mg} / \mathrm{k},{ }^{10}$ inferior a la usada por vía intramuscular ${ }^{11}$ y cercana a la de la vía intravenosa. No existen estudios sistemáticos que hayan evaluado la SSL como relajante muscular con relación de otras vías. El objetivo de este estudio fue comparar la eficacia de la SSL y la SIM con la de la SIV, como relajante muscular en adultos sometidos a cirugía electiva, para extrapolar la información obtenida a la condición clínica del evento potencialmente catastrófico de laringoespasmo en ausencia de acceso venoso.

\section{Materiales y métodos}

Se diseñó un ensayo clínico en el que pacientes mayores de edad programados para cirugía electiva y cuyo estado físico fuera clasificado como pacientes adultos de bajo riesgo (ASA I o II), aceptaban por escrito participar. Después se asignaban al azar para inducción con SSL, SIM, o SIV. Se excluyeron del estudio los pacientes con antecedentes de alergia a la succinilcolina o cruzada con relajantes neuromusculares, historia personal o familiar de hipertermia maligna, enfermedades neurológicas de base, miopatías, distrofias musculares, inmovilización prolongada, hipertensión endocraneana, historia de glaucoma, antecedentes personales o familiares de presencia de variantes de colinesterasa plasmática, embarazo, edad mayor de 60 años, tratamiento con neostigmina, fisostigmina, propranolol, esmolol, digitálicos, quinidina, corticoides, anticonceptivos orales, citotóxicos y anticancerosos.
Se empleó un muestreo por conveniencia. Se calculó el tamaño de la muestra necesaria de 15 pacientes en cada grupo, asumiendo un alfa de 0.05 , un beta de 0,2 y una diferencia estimada en tiempo de relajación de 195 segundos.

Las dosis de succinilcolina utilizadas fueron: 1 $\mathrm{mg} / \mathrm{k}$ de SSL, $5 \mathrm{mg} / \mathrm{k}$ de SIM y $1 \mathrm{mg} / \mathrm{k}$ de SIV. Previa calibración de instrumentos, el anestesiólogo valoró la contracción muscular utilizando el estimulador TOF Watch $^{\circledR}$, y mediante cronómetro determinó los tiempos de latencia, acción y recuperación. Se definió como latencia, el tiempo en minutos desde la aplicación hasta el inicio de la acción relajante, evidenciada por pérdida del estímulo simple de tren de cuatro (prueba de contracción muscular utilizando cuatro estímulos). Se definió la acción (duración) como el tiempo en minutos desde el inicio de la relajación hasta la terminación de la acción relajante, evidenciada por la recuperación del primer estímulo del tren de cuatro. Se definió la recuperación como el tiempo en minutos desde el inicio de primer estimulo del tren de cuatro hasta la recuperación completa de la contracción muscular, evidenciada por la aparición de los cuatro estímulos del tren de cuatro. Adicionalmente se registró para cada paciente la edad (Tabla 1), el estado físico (puntaje ASA) y el género.

Los datos recolectados se tabularon en el software Microsoft Excel. Se realizó una descripción de las variables utilizando medidas de frecuencia o de tendencia central y dispersión según el tipo de variable. Además, se compararon los efectos de las vías empleadas mediante la prueba exacta de Fisher (categorizando tiempo de latencia en mayor de diez minutos y menor o igual a diez minutos, y tiempo de acción en mayor de cuatro y menor o igual a cuatro minutos), y la prueba de Mann-Whitney utilizando las variables dependientes como números expresados en segundos (tiempo de latencia) y minutos (tiempo de acción). Los análisis se realizaron mediante el software estadístico SAS versión 8.2. Se consideraron como significativos los valores de $p$ menores a 0.05 .

El estudio fue aprobado por los comités institucionales de investigación y ética. 


\begin{tabular}{|c|c|c|c|c|}
\hline \multicolumn{5}{|c|}{$\begin{array}{l}\text { Tabla I.Tiempos de latencia, acción y recuperación } \\
\text { según la vía de administración de la succinilcolina. }\end{array}$} \\
\hline \multicolumn{2}{|c|}{ Tiempos } & SIV & SIM & SSL* \\
\hline $\begin{array}{l}\text { T. latencia } \\
\text { (minutos) }\end{array}$ & $\begin{array}{l}\text { Media (Des. est) } \\
\text { Mediana (Rango) }\end{array}$ & $\begin{array}{c}0.68(+/-0.07) \\
0.66(0.58-0.76)\end{array}$ & $\begin{array}{c}4(+/-1.22) \\
4(3-6)\end{array}$ & $\begin{array}{l}9(+/-1) \\
9(8-10)\end{array}$ \\
\hline $\begin{array}{l}\text { T. acción } \\
\text { (minutos) }\end{array}$ & $\begin{array}{l}\text { Media (Des. est) } \\
\text { Mediana (Rango) }\end{array}$ & $\begin{array}{c}6.2(+/-1.48) \\
6(4-8)\end{array}$ & $\begin{array}{c}5.6(+/-|.5|) \\
5(4-8)\end{array}$ & $\begin{array}{l}3(+/-1) \\
3(2-4)\end{array}$ \\
\hline $\begin{array}{l}\text { T. recuperación } \\
\text { (minutos) }\end{array}$ & $\begin{array}{l}\text { Media (Des. est) } \\
\text { Mediana (Rango) }\end{array}$ & $\begin{array}{c}8.6(+/-1.94) \\
8(6-11)\end{array}$ & $\begin{array}{c}7.8(+/-3.27) \\
8(3-12)\end{array}$ & $\begin{array}{l}4.5(+/-1.5) \\
4.5(3-6)\end{array}$ \\
\hline
\end{tabular}

Des. est: Desviación estándar.

*Solo dos pacientes lograron relajación muscular con la administración sublingual.

\section{Resultados}

El total de pacientes fue de 15, de los cuales cinco se asignaron en forma aleatoria a la vía intravenosa, cinco a la intramuscular y cinco a la sublingual. La inclusión de más pacientes se detuvo pues con un número limitado de observaciones fue evidente que la administración sublingual daba resultados inferiores a las otras vías.

La edad promedio de los pacientes fue de 39.6 años (rango 27-61). De los quince, doce fueron mujeres $(80 \%)$ y tres fueron hombres $(20 \%)$, para una relación de 4 a 1 . El porcentaje de enfermos en ASA I fue de 60\% (9) y en ASA II fue de $40 \%$ (6).

Solo dos pacientes a quienes se les administró SSL alcanzaron relajación muscular. El tiempo de latencia fue significativamente menor en pacientes en quienes se utilizó la SIV en comparación con la SIM (mediana de 42 segundos vs. 240 segundos, $p=0.03$ ). El tiempo de acción fue más prolongado en quienes se usó SIV vs. SIM, pero esta diferencia no fue significativa (mediana seis minutos $v s$. cinco minutos, $p=0.5$ ).

Un paciente en el grupo de SSL alcanzó tiempos de latencia inferior a diez minutos y de acción su- perior a dos minutos, en comparación con el 100\% para aquellos con SIM o SIV, $(p=0.02)$. Ninguno presentó efectos adversos.

\section{Discusión}

Nuestro estudio demuestra que los tiempos de latencia y de acción de la SSL a dosis de $1 \mathrm{mg} / \mathrm{k}$ en adultos, no son adecuados comparados con la SIM o SIV. Consideramos que la razón para la ineficacia de la SSL puede explicarse por una dosis insuficiente.

La única estructura que se encuentra debajo de la mucosa del piso de la boca es el músculo milohioideo, lo cual nos permite teorizar que la dosis sublingual debe ser cercana a la intramuscular. Sin embargo, esta última es cinco veces la utilizada en nuestro estudio para la vía sublingual y la inyección del volumen correspondiente a esa dosis sería difícil desde el punto de vista técnico e incrementaría los riesgos de complicaciones locales como hematomas, dolor y sangrado. Debido a la rica vascularización de la zona, también existiría el peligro de inyección intravascular involuntaria, lo cual podría aumentar el riesgo de complicaciones sistémicas como arritmias, reacciones miotónicas, hipercalemia y aumento de las presiones intracraneana e intraocular. 
Aún con el limitado número de pacientes, nuestros resultados indujeron la suspensión del estudio en más pacientes. De desearse una iniciativa investigativa adicional, consideramos pertinente estudiar más en detalle la farmacocinética y farmacodinamia de la SSL, de preferencia en animales de experimentación, para después extrapolar dicha información con precaución a estudios en humanos.

\section{Agradecimientos}

A los residentes e instructores de anestesiología de la Fundación Universitaria de Ciencias de la Salud, Hospital de San José, por su colaboración durante la selección de los pacientes, al Dr. Álvaro Sanabria, ex-miembro de la División de Investigaciones Fundación Universitaria de Ciencias de la Salud, por la orientación metodológica inicial del proyecto.

\section{Referencias}

1. Landsman IS. Mechanisms and treatment of laryngospasm. Int Anesthesiol Clin. 1997; 35(3):67-73.

2. Murray D. Prep reparation of succinylcholine. Anesthesia. 2004;59(8):823-4.
3. Chung DC, Rowbottom SJ. A very small dose of suxamethonium relieves laryngospasm. Anaesthesia. 1993;48(3): 229-30.

4. Fodale V, Pratico C, Leto G, Caminiti V, Mazzeo AC, Lucanto T. Propofol relieves post-extubation laryngospasm in obstetric anesthesia. Int J Obstet Anesth. 2004;13(3):196-7.

5. Moore JP, Pace SA, Busby W. Comparison of intraosseous, intramuscular, and intravenous administration of succinylcholine. Pediatr Emerg Care. 1989;5(4):209-10.

6. Seah TJ, Chin NM. Severe laryngospasm without intravenous access a case report and literature review of the non-intravenous routes of administration of suxamethonium. Singapore Med J. 1998;39(7):328-30.

7. Warner DO. Intramuscular succinylcholine and laryngospasm. Anesthesiology. 2001;95(4):1039-40).

8. Schuh FT. The neuromuscular blocking action of suxamethonium following intravenous and intramuscular administration. Int J Clin Pharmacol Ther Toxicol. 1982;20(9):399-403.

9. Redden RJ, Miller M, Campbell RL. Submental administration of succinylcholine in children. Anesth Prog. 1990;37(6): 296-300.

10. Mazze RI, Dunbar RW. Intralingual succinylcholine administration in children: an alternative to intravenous and intramuscular routes? Anesth Analg. 1968;47(5):605-15.

11. Sutherland GA, Bevan JC, Bevan DR. Neuromuscular blockade in infants following intramuscular succinylcholine in two or five per cent concentration. Can Anaesth Soc J. 1983;30(4):342-6.
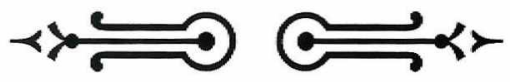